\title{
Modelling and design of optimized electronic wedge brake
}

\begin{abstract}
This paper discusses the modelling and design of Electronic Wedge Brake (EWB) for a typical passenger car. The optimized version of EWB used as a brake mechanism while Permanent Magnet DC Motor is used as an actuator to drive the mechanism. Complete optimized EWB mathematical modelling is derived from PMDC Motor, leadscrew with gear and EWB based brake heart. The wedge angle is set based on the disk pad coefficient and motor selection is done based on brake design requirement. Simulation is performed to verify the performance of the designed EWB brake. The EWB brake can be described as a linear model with 5th order system in state space form. The design requirement for the brake should be clear to ensure a newly designed EWB based brake perform well accordingly.
\end{abstract}

Keyword: Optimized electronic wedge brake (EWB); Modelling; Permanent magnet DC motor (PMDC) 